\title{
Percepciones del estudiantado, discriminación educacional por medio de la normalización de la exclusión
}

Perception of students; scholar discrimination through exclusion standardization

Oñate Escobar, Susana Arlette. y Alfaro Urrutia, Jorge Eduardo. (2021). Percepciones del estudiantado, discriminación educacional por medio de la normalización de la exclusión. Revista Actualidades Investigativas en Educación, 21(3), 1-27. Doi. 10.15517/aie.v21i3.46483 


\title{
Percepciones del estudiantado, discriminación educacional por medio de la normalización de la exclusión
}

Perception of students; scholar discrimination through exclusion standardization

\author{
Susana Arlette Oñate Escobar ${ }^{1}$ \\ Jorge Eduardo Alfaro Urrutia²
}

Resumen: La educación cumple un rol esencial en la formación de una sociedad más justa. Chile es un país en que las políticas de inclusión educativa trabajan de manera paralela a las políticas de integración. Por ello es fundamental develar cómo se presentan las prácticas de exclusión, segregación y discriminación en escuelas, con el fin de estudiar cómo obstaculizan la inclusión. Este artículo expone los resultados de un estudio que se sustenta en el paradigma interpretativo, un diseño cualitativo y un caso único de investigación. Se desarrolla en el año 2019 , con la participación de 8 estudiantes, en un establecimiento escolar con gran trayectoria y características que lo hacen interesante de conocer, en la comuna de Temuco, región de La Araucanía, Chile. Esto permite analizar y categorizar las percepciones del estudiantado sobre prácticas de respuesta a la diversidad. Los resultados de investigación arrojaron percepciones sobre prácticas de respuesta excluyentes, segregadoras, de integración e inclusivas, esta última en tres subcategorías: relaciones, estrategias pedagógicas y políticas inclusivas. La discusión muestra que las prácticas de exclusión son normalizadas y justificadas cuando estas no afectan directamente al grupo dominante o bien, cuando la inclusión puede perjudicar el rendimiento individual de las personas pertenecientes a ese grupo. Asimismo, se expone que esta comunidad educativa se enfoca en la promoción de una cultura inclusiva, sin cuestionar la estructura normativa formal de respuestas a la diversidad, lo que genera que el estudiantado perciba prácticas segregadoras frente a Necesidades Educativas Especiales.

Palabras clave: educación inclusiva, discriminación educacional, agrupamiento educacional.

Abstract: Education plays an essential role in the formation of a more just society. In Chile, inclusive policies coexist with integration education policies. Therefore there is a need to reveal how exclusion, segregative and discriminative practices are presented in schools in order to understand how this processes hinder inclusion. This article presents the results of an interpretive paradigm based study, a qualitative design and a case study research. It takes place in 2019, with the participation of 8 students, in a school with long history and characteristics that make it interesting to know in Temuco City, region La Araucania, Chile. The study analyze and categorize students perceptions about school respond to diversity practices. The research results yielded perceptions about exclusive segregating, integrative and inclusive responde practices, the latter in three categories: relationships, pedagogical strategies and inclusive polices. Discussion shows that exclusion practices are normalized and justified when they do not directly affect the dominant group or, when inclusion can harm the individual performance of the people belonging that group.

Keywords: inclusive education, scholar discrimination, scholar group.

\footnotetext{
${ }^{1}$ Universidad de la Frontera, Programa Magíster en Educación, Temuco, Chile. Dirección electrónica: susanaaoe@gmail.com Orcid https://orcid.org/0000-0001-6364-854X

2 Universidad de la Frontera, Programa Magíster en Educación, Temuco, Chile. Dirección electrónica: Jorgeeduardoau@gmail.com Orcid http://orcid.org/0000-0003-3711-6536
}

Artículo recibido: 30 de marzo, 2021

Enviado a corrección: 4 de junio, 2021

Aprobado: 16 de agosto, 2021 


\section{Introducción}

El rol formativo de la escuela es esencial para cumplir el desafío de construir una sociedad más justa y pacífica. Es por ello que valores como la tolerancia, respeto y pluralismo son primordiales en la educación de niños, niñas y jóvenes (Unesco, 2011). Sin estos valores no es posible llevar a cabo una convivencia escolar positiva que prepare al alumnado para la vida en una sociedad inclusiva. Así, el rol formativo de la educación provoca que sea necesario comprender las dinámicas de exclusión, segregación y discriminación que se llevan a cabo en la escuela, con el fin de desarticular las barreras de inclusión presentes en esta (Ramírez, 2017) y que impiden que todo el alumnado sea un participante activo de la comunidad. Al obtener claridad sobre estas dinámicas escolares es posible responder de manera más efectiva a la diversidad presente en el aula (Rittaco y Amores, 2012).

En Chile, las políticas nacionales han buscado avanzar en la atención de la diversidad. Prueba de este esfuerzo, son el cambio de una Ley de Integración (Ley 19.284, 1994) a una Ley de Inclusión Social e Igualdad de Oportunidades (Ley 20.422, 2008), así como la incorporación de Leyes, como la de Inclusión Escolar (Ley 20.845, 2015) y Decretos específicos para responder a Necesidades Educativas Especiales (NEE) (Decreto 170, 2009; Decreto 83, 2015). A pesar de estos esfuerzos por diversificar la enseñanza y equiparar las oportunidades para todos y todas, la necesidad de obtener resultados en pruebas estandarizadas chilenas permea todos los actores de una comunidad educativa (NavarroAburto, Arriagada, Osses-Bustingorry y Burgos-Videla, 2016). Es así como, por ejemplo, el Sistema de medición de la calidad de la educación (SIMCE) y la Prueba de selección universitaria (PSU) obstaculizan los avances para flexibilizar la forma en que se brinda la educación al alumnado (Inostroza y Falabella, 2021). En este sentido, el enfoque neoliberal y económico del modelo educativo chileno (Flórez, 2015; Queupil y Durán, 2018) es incoherente con la necesidad de tener un modelo flexible que responda a la diversidad.

Aunque existen barreras institucionales, no son pocas las recientes experiencias chilenas que destacan avances y alertan sobre facilitadores internos en la implementación de una educación inclusiva (Barría, 2019; Figueroa-Céspedes, Soto y Yáñez-Urbina, 2019; Poblete-Christie, López y Muñoz, 2019). En este estudio se plantea el objetivo de evaluar la percepción del estudiantado respecto de las prácticas de respuesta a la diversidad, que facilitan u obstaculizan el desarrollo de una comunidad inclusiva. Lo anterior, con el fin de proponer nuevas prácticas inclusivas. Por eso, los objetivos específicos de la investigación se 
enfocan en la identificación, análisis y categorización de las respuestas a la diversidad que la comunidad ejerce, a partir de las percepciones del estudiantado.

Este proceso de investigación pudo realizarse gracias al financiamiento de la Agencia Nacional de Investigación y Desarrollo (ANID), por medio de la obtención de la Beca de Magíster en Chile para Profesionales de la Educación, adjudicada el año 2018. La pregunta que ha guiado el proceso de investigación es: ¿Cuáles son las percepciones del estudiantado respecto de las prácticas de respuesta a la diversidad, que facilitan u obstaculizan el desarrollo de una comunidad inclusiva?

\section{Referentes teóricos}

\subsection{Formas de respuesta a la diversidad}

Son varios los estudios que han abordado cómo los seres humanos nos enfrentamos a la diversidad en el contexto escolar. Por ejemplo, Escribano y Martínez (2013) plantean que la educación inclusiva es una forma de respuesta a la diversidad que tiene como principal objetivo eliminar la exclusión. También explican que se entiende por exclusión toda acción que niegue la participación plena del alumnado en su comunidad educativa. Además, cuando el estudiantado experimenta barreras de acceso o participación de la vida escolar, esto perjudica su aprendizaje y desarrollo, lo que profundiza las desigualdades sociales.

Otra forma de responder a la diversidad es la segregación, la que se reconoce como el acto de separar grupos humanos de acuerdo con sus características, ya sea por nivel socioeconómico, rendimiento, género u otros. Ello tiene un impacto negativo en el aprendizaje del estudiantado (Núñez-Muñoz, Peña-Ochoa, González-Niculcar y Ascorra-Costa, 2020), sus expectativas y motivación, especialmente en grupos vulnerables (Murillo y Duk, 2016). Este es un factor relevante como causa del abandono escolar (Vázquez-Recio y López-Gil, 2018).

Finalmente, la integración escolar es definida por Escribano y Martínez (2013) como la incorporación de una diversidad de estudiantes al sistema educativo regular, pero enfocada en la búsqueda de su normalización. De esta forma, se propicia que el alumnado se adapte al sistema escolar, lo que se contrapone con una respuesta inclusiva en que la escuela es la que debe ser flexibilizada para responder a todos sus miembros (Véliz, Martínez, Parra y Garrido, 2020). 


\subsection{Facilitadores de inclusión}

A partir de la publicación de diversas experiencias de comunidades que buscan ser más inclusivas, se destacan algunas características comunes a nivel internacional y nacional. La primera de ellas es asumir la educación inclusiva como un fenómeno complejo. Esto permite aceptar que se trata de un proceso siempre en movimiento, que requiere de una deconstrucción de prácticas de exclusión para una construcción de nuevas prácticas inclusivas (Escribano y Martínez, 2013; Sinisi, 2010). Se trata, por tanto, de un ejercicio de observación, reflexión, evaluación y creación constante por parte de quienes gestionan la escuela (Parra Muñoz, et al., 2020). Este ejercicio de observación es caracterizado como "la confianza básica, la actitud expectante y la actitud amorosa; dinamismos que están en la base de la persona" (Escribano y Martínez, 2013, p. 36). El desarrollo de estas características permite mostrar una apertura en la cultura inclusiva, pues serían fundamentales para poder identificar prácticas y experiencias de exclusión.

Otro de los factores que han tomado fuerza en el desarrollo de una escuela inclusiva, es la incorporación de diseños universales de aprendizaje. Esta forma de concebir la organización curricular representa un facilitador, pues toma en cuenta a todo el alumnado durante la planificación, al responder a una mirada compleja de la educación, y generar un estado de equidad para el logro de objetivos (Gimeno Sacristán, 2011; Ortíz, López, Figueredo y Martín, 2018). Al considerar múltiples medios de representación, expresión y compromiso (Martín y Mauri, 2011), estas planificaciones trabajan en la búsqueda de posibilidades que faciliten el desarrollo del estudiantado. Además, los planteamientos de este diseño curricular invitan al profesorado a aprovechar los medios tecnológicos disponibles (Alba, 2015), lo que puede eliminar barreras que las estrategias, herramientas y materiales pedagógicos tradicionales implican.

Muy asociado a esta forma de planificar el aprendizaje se encuentran las estructuras cooperativas de trabajo en el aula (Escribano y Martínez, 2013; Gimeno Sacristán, 2011; Ortíz, et al., 2018; Torrego, Monge, Pedrajas y Martínez, 2015). Estas son aquellas en que existe interdependencia positiva de metas, recompensas, recursos o funciones (Johnson y Johnson, 2018). Este tipo de organización cooperativa de tareas escolares tiene un gran impacto en la convivencia escolar positiva, al otorgar al alumnado una instancia de aprendizaje heterogéneo que ayuda a salvaguardar sus diferencias y potenciar habilidades individuales.

La razón detrás de los buenos resultados de las tareas realizadas cooperativamente es explicada por Johnson y Johnson (2018). Los autores mencionan que frente a la existencia de 
una estructura de interdependencia, cada miembro del grupo de trabajo aumenta el esfuerzo que realiza por conseguir buenos resultados, en comparación con otras instancias de carácter individual o competitivo. Esto también ocurre en el esfuerzo por mantener una relación interpersonal agradable y sentirse en un estado de salud mental positivo (Johnson y Johnson, 2018).

A nivel latinoamericano, Muñoz (2018) destaca y sintetiza una serie de elementos comunes en experiencias positivas de educación inclusiva. Uno de ellos es la valoración del alumnado y su contexto, acompañado del desarrollo de currículos flexibles y basados en habilidades para la vida. Del mismo modo, se observa una mayor apropiación de una cultura inclusiva en instituciones que han realizado procesos de sensibilización. Finalmente, en un ámbito distinto, menciona como elementos comunes el trabajo colaborativo entre profesores y estudiantes, el ejercicio del liderazgo participativo y una alta implicación del alumnado en organizaciones escolares (Muñoz, 2018).

A partir del conocimiento de las experiencias anteriores, resulta interesante profundizar en las percepciones del alumnado respecto de las formas de respuesta a la diversidad que se ejercen en su comunidad. Pues, si bien, múltiples experiencias internacionales coinciden en elementos facilitadores de una comunidad inclusiva (Casanova, 2020; Escribano y Martínez, 2013; Grácia, Sanlorien y Segués, 2017; Hernández, 2016; Johnson y Johnson, 2018; López Melero, 2018; Moliner, 2013; Muñoz, 2018; Murillo y Duk, 2016; Ortíz, et al., 2018), estos se conocen a partir de las experiencias del profesorado, equipos de gestión y comunidad universitaria.

\section{Marco metodológico}

\subsection{Enfoque}

La presente investigación se enmarca en un enfoque cualitativo de investigación, con un diseño de estudio de caso único (Mayan, 2001). Esto, pues, se explora en las prácticas de respuesta a la diversidad, en una institución educativa de la comuna de Temuco, Chile, en el año 2019. Ello, con el fin de realizar un análisis profundo y detallado del fenómeno en estudio, en un establecimiento con una trayectoria y proyecto educativo que se declara inclusivo. Asimismo, la investigación se sustenta en el paradigma interpretativo, ya que busca profundizar en las percepciones sobre prácticas de respuesta a la diversidad que apoyan y desfavorecen la educación inclusiva. Esto, por medio del análisis de la construcción que los 
sujetos hacen de dichas prácticas a través de sus experiencias (González-Monteagudo, 2000; Mella y Osses, 2015).

\subsection{Unidades de análisis}

Las personas participantes del estudio corresponden a estudiantes de último año de escolaridad con trayectoria escolar completa. Por tanto, ingresaron al establecimiento en primer año de educación básica y cursan, en el momento del contacto, el último año de enseñanza media. Esto quiere decir que han estudiado al menos 12 años en la institución. Se decidió este criterio para poder tener la percepción de estudiantes que han pasado por experiencias, cambios y docentes en la institución.

De una preselección de 57 estudiantes, que cumplieron con los criterios antes señalados, se trabajó finalmente con un total de 8 estudiantes que participaron voluntariamente, 4 hombres y 4 mujeres de entre 17 y 19 años. El estudiantado cursaba el segundo semestre de cuarto año medio al momento del contacto. Asimismo, conformaron un grupo que se caracterizó por tener rendimientos escolares variados, provenir de localidades rurales y urbanas, ser estudiantes con y sin discapacidad, migrantes y no migrantes y contar un miembro de la comunidad LGBT.

Para llegar al grupo de participantes, se solicitó autorización a la dirección del establecimiento para que docentes con jefatura del nivel de cuarto año medio informaran sobre el estudiantado que cumplía con el criterio de trayectoria completa. El alumnado que voluntariamente accedió a participar recibió un protocolo de consentimiento y asentimiento que detallan el carácter confidencial y anónimo de su participación. Todas las personas seleccionadas aceptaron participar de la investigación. En el caso de estudiantes menores de 18 años, el o la apoderada también consintió la participación. Este periodo de contacto duró 8 semanas.

El establecimiento educativo en el que se realizó la investigación cuenta con 116 años de ejercicio en la docencia, con una matrícula total de 3949 personas matriculadas entre nivel de transición uno y cuarto año de enseñanza media. Además de un equipo docente de 179 personas y 198 asistentes de la educación, que ejercen múltiples labores que apoyan la gestión de la institución. Con un índice de vulnerabilidad aproximado de un 86\%, esta institución cuenta con un Programa de Integración Escolar (PIE) desde el año 2005 que, a la fecha, atiende a un total de 468 estudiantes de todos los niveles educativos que imparte, con un $11,85 \%$ del total de la población escolar. Esta cantidad de estudiantes lo convierte en el 
colegio con el Programa de Integración Escolar más grande del país y un proyecto educativo institucional que expresa un enfoque de educación inclusiva.

\subsection{Técnicas de recolección}

Se aplicaron dos instrumentos, una entrevista semiestructurada en profundidad y un grupo focal (Gurdián-Fernández, 2007), ambos previamente evaluados por un equipo experto. La entrevista se realizó con veintidós preguntas al estudiantado, organizadas en torno a dos temas: prácticas de respuesta a la diversidad ejercidas por docentes dentro del aula y prácticas de respuesta a la diversidad ejercidas fuera del aula. Una vez analizada cada entrevista se le solicitó al estudiantado participante que observen, cuestionen y aprueben el análisis realizado como estrategia de verificación, con el fin de asegurar la validez y el rigor de la investigación (Mayan, 2001).

Finalizadas las entrevistas se propuso un grupo focal de 6 preguntas, las que buscaron profundizar en las percepciones del alumnado sobre diversidad e inclusión, así como de otros conceptos complementarios que surgieron de las primeras entrevistas, tales como convivencia, comunidad y justicia. Cuatro estudiantes accedieron a participar de este procedimiento.

\subsection{Procesamiento de análisis}

Las entrevistas y grupo focal fueron grabadas y transcritas textualmente para iniciar el proceso de categorización (Dávila, 2006). Se procedió a identificar las ideas que el estudiantado reconociera como relacionadas con la forma en que el establecimiento responde a cualquier tipo de diversidad. Estas respuestas se agruparon en categorías que, a su vez, se clasificaron según fueran valores, conceptos o estrategias para conformar subcategorías. Se analizó cuáles eran las finalidades de las estrategias y los motivos de los valores, así como la conexión entre estos.

La codificación fue realizada de manera abierta y axial. La primera consistió en identificar conceptos a partir de ciertas características y propiedades, mientras que la segunda consistió en el establecimiento de relaciones y reagrupaciones en función de dichas características (Strauss y Corbin, 2012). El software utilizado para ello fue Atlas Ti, en su séptima versión. 


\section{Resultados principales y discusión}

A continuación, se presentan los resultados de la investigación a partir de la identificación de las percepciones sobre las respuestas a la diversidad que el alumnado ha identificado a través de sus relatos. De esta forma, también se analizan y categorizan en cuatro prácticas, estrategias y actitudes diferentes de respuesta a la diversidad en esta comunidad educativa, para cumplir los objetivos específicos del estudio. Estos fueron: 1. Identificar la percepción del estudiantado respecto de las prácticas de respuesta a la diversidad que ejerce el equipo docente y administrativo pertenecientes a una comunidad educativa. 2. Analizar las características de las prácticas de respuesta a la diversidad en una comunidad educativa desde la perspectiva del estudiantado. 3. Categorizar las prácticas de respuesta a la diversidad en una comunidad educativa desde la perspectiva del estudiantado. 4. Proponer, a partir de los resultados obtenidos, prácticas inclusivas de respuesta a la diversidad para la comunidad educativa.

Para ello se van intercalando los resultados, discusión y reflexiones en torno a los dos apartados expuestos a continuación, los cuales representan la categorización de las prácticas percibidas por el alumnado. El objetivo específico número 4, se expresa en las conclusiones de esta investigación. De esta forma, los resultados se enfocaron en demostrar que cualquiera que sea la percepción del estudiantado respecto de la diversidad, esta se encontrará posicionada sobre un conjunto de valores que determinarán formas de acción. Estos valores serán sensibles a medidas pedagógicas, como la relación entre profesorado, alumnado y evaluación.

\subsection{Prácticas, estrategias y actitudes inclusivas}

Los resultados permiten identificar múltiples prácticas inclusivas de respuesta a la diversidad, enfocadas hacia la promoción de valores, estrategias pedagógicas y políticas de acceso. El estudiantado participante del estudio expone estas prácticas sin excepción, lo que devela un proceso que la institución educativa ha llevado a cabo con el fin de responder a su proyecto educativo.

El alumnado reconoce la pertenencia a una comunidad educativa que es más inclusiva que la sociedad, por lo que comparan constantemente entre lo que ellos consideran un mundo interno y uno externo, dentro y fuera de su colegio (ver cita 1 y 2 ). Fuera del establecimiento, reconocen que la discriminación es la respuesta más común a la diversidad, por lo que expresan admiración y sorpresa de que el profesorado no ejerza dicha práctica. 
Cita 1: "Yo creo si, por ejemplo en comparación con otros colegios, como que este colegio es más diverso, como que tiene más información sobre ciertas cosas, y como que en algunos profes son súper actualizados, digo, hay algunos profes que son más viejos que otros (risas). Hay de todo, pero igual hasta los profes más viejos, toman súper bien la diversidad aquí." (Entrevista 5, P.6)

Cita 2: "yo creo que mis compañeros son como, que donde somos todos tan diferentes no les causa como una mayor intriga al ver a alguien diferente, diciendo "éste es de esta forma y de esta forma", sino que lo aceptan." (Entrevista 6, P.3)

Por otra parte, prácticas inclusivas de respuesta a la diversidad económica, como la adscripción de la institución a la gratuidad educativa, es percibida por el estudiantado como una oportunidad de convivir con sus pares pertenecientes a diversos niveles socioeconómicos (ver cita 3 y 4$)$.

Cita 3: "creo que hay bastantes alumnos y de todo tipo, en parte porque si no me equivoco, es gratis el colegio. Eso ya abre más posibilidades a que entre gente diversa" (Entrevista 8, P. 4)

Cita 4: "igual lo que me gusta del colegio es que hay todo tipo de gente, yo pensaba que por ser gratis iba a haber solo gente como uno, pero como estamos entre dos ciudades igual como que al final tengo compañeros de todo tipo" (Entrevista 3, P.5)

Al analizar estas percepciones se han categorizado como prácticas inclusivas aquellas que involucran valores, estrategias y políticas que facilitan, promocionan o aseguran el acceso y la participación de todo el alumnado dentro y fuera de la sala de clases (Escribano y Martínez, 2013; Hernández, 2016).

Del mismo modo, prácticas inclusivas como la presencia de múltiples actividades complementarias, estrategias cooperativas de construcción del conocimiento, la variabilidad en las estrategias de enseñanza-aprendizaje, entre otras, son valoradas positivamente por el estudiantado participante del estudio, pues les permiten ser miembros activos de la comunidad (ver cita 5 y 6), también como facilitadoras de participación (Escribano y Martínez, 2013). 
Cita 5: "Yo... Bueno, este año he notado que hay como más...actividades extraescolares, hay que todo. También que en clases no es como solamente pruebas, sino que hay más como trabajo al aire libre, o videos, o hasta hacer una canción sobre alguna materia, esas cosas son más entretenidas y trabajamos en equipo, igual las profes tratan de que podamos como hacer de todo". (Entrevista 5, P. 8)

Cita 6: "Igual los profesores tratan de hacer eso como para fomentar el compañerismo y el pensar en ello, porque también más adelante uno también tiene que hacer trabajos grupales o trabajar con otras personas alrededor y aprender a convivir." (Entrevista 3, P.2).

La promoción de valores inclusivos aparece también como una idea que destaca el estudiantado, pues le hace sentir que la relación con sus docentes se da en un entorno de empatía, comprensión, acogida, solidaridad, justicia y valoración de la diversidad, entre otros valores facilitadores de la inclusión y el sentido de comunidad (ver cita 7 y 8 ).

Cita 7: En las evaluaciones, a veces por ejemplo cuando te ven como muy pensativo y como muy frustrado quizás con la respuesta, después, algunos profes me han preguntado. Ya ¿qué te pasa?, ¿cómo estay?, ¿te ha pasado algo en tu casa o algo?; porque tienen como ese, no sé, cómo ese instinto de saber que algo le pasa a sus alumnos. (Entrevista 4., P.1)

Cita 8: "Cuando tenemos algún problema, ellos nos ayudan, nos abrazan en un sentido, nos dan consejo, el profe jefe es el que más cercano, que uno quiere conocer. Se preocupa, habla con los apoderados." (Entrevista 1, P.1)

Este sentido de pertenencia que el alumnado manifiesta en sus relatos, producto de prácticas, actitudes y estrategias inclusivas, es importante, pues permite el desarrollo de una comunidad cohesionada con objetivos comunes. La cohesión social y el sentido de pertenencia a la comunidad disminuyen las posibilidades de exclusión, por cuanto los participantes del grupo sienten motivación por ejercer una ciudadanía activa, a la vez que aumentan los lazos entre los miembros de la sociedad (Castillo Riquelme, Rodríguez Garcés y Escalona Burgos, 2018; Naciones Unidas, Cepal, 2007). La importancia de la cohesión social 
es extrapolable a las comunidades educativas, pues estas últimas se fundan en proyectos institucionales que necesitan de toda la comunidad para el logro de sus objetivos.

A pesar del avance que el estudiantado percibe, resulta interesante que en sus relatos se encuentran ausentes percepciones respecto de acciones de discusión, promoción y valoración que permitan visibilizar la diversidad. Esta ausencia puede evidenciarse en el hecho de que, en los resultados de la investigación, el alumnado no percibe las diversidades étnicas, de capacidades, sociales y económicas presentes en su comunidad, aun cuando entrevistados se identifican con esos grupos. En cambio, sí perciben las diversidades de género y nacionalidad. (ver cita 9 y 10).

Cita 9: "Es que casi no se ven aquí, se ven más adultos que son inmigrantes Haitianos, y yo cacho que en unos años más, como he visto embarazadas, ahí se van a ver más...Por eso no hay tantos adolescentes todavía." (Entrevista 7, P. 5)

Cita 10: "A si, ahora sí que nada que decir de los profes, no hay, nunca ha tocado como un caso de un profesor que haya hecho sentir mal a un... a una persona, por sus gustos o cosas. Mi curso igual de hecho es como, hay hartas personas que son homosexuales, y mis profesores como que no, ya es como tan normal que no es tema ya." (Entrevista 2, P. 3)

Esto ocurre porque las prácticas de respuesta a la diversidad en el establecimiento tienen su principal foco en la promoción de valores inclusivos, que devienen en acciones que alientan la participación del alumnado y buscan asegurar su acceso a la educación, sin embargo, no visibilizan, valoran, ni destacan la diversidad directamente (Vega Fuente, López Torrijo y Garín Casares, 2013). Hablar de las diversidades presentes en la comunidad es necesario para llevar a cabo un análisis consciente de los elementos que es necesario modificar, cambiar o modernizar para hacer de la educación una experiencia de equidad e inclusión.

Es posible inferir que, a raíz de la falta de diálogo, análisis y reflexión en torno a la diversidad presente en la comunidad, tampoco se cuestiona la estructura formal de las respuestas a la diversidad que ejercen, especialmente cuando estas se encuentran sustentadas en una normativa legal del macro sistema educativo. Esto queda en evidencia cuando se les pregunta al estudiantado sobre los apoyos educativos que perciben (ver cita 11 y 12) en el siguiente apartado. 
La percepción del alumnado respecto de la diversidad se estaría orientando hacia una comunidad educativa que parece un lugar seguro para la expresión de la propia identidad y que es acogedora con las diferencias. Sin embargo, la percepción de la diversidad no incluye en la discusión las prácticas o valores de exclusión, segregación e integración. Esto permite que tales prácticas se justifiquen y normalicen. Por tal motivo, en el siguiente apartado se demostrará que en una comunidad educativa, compuesta de muchos valores y prácticas respetuosos de la diversidad, mientras no se cuente con mecanismos de identificación y discusión de la exclusión, segregación y discriminación se adosarán prácticas excluyentes que no serán percibidas por sus integrantes.

\subsection{Prácticas, estrategias y actitudes excluyentes, segregadoras e integradoras}

En la configuración de los cursos del establecimiento existe un grupo mayoritario de estudiantes que parece lograr un desempeño escolar acorde a las expectativas que se les plantean. El estudiantado entrevistado identifica la existencia de dos grupos, uno mayoritario que parece lograr un desempeño escolar acorde a las expectativas planteadas, y otro grupo minoritario que es percibido con características negativas, que impiden que cumplan con las exigencias del sistema educativo en el que se encuentran insertos.

Una primera manifestación de la atribución de características negativas a quienes no cumplen con expectativas académicas o de comportamiento puede observarse en las citas 11 y 12 expuestas a continuación.

Cita 11: “...sé que a chicos que les cuesta más o una actividad, les hacen forma $C$ y las rinden con una educadora diferencial, como para poder reforzarlos más en esa área." (Entrevista 3, P.3)

Cita 12: “...cuando chico me mandaban casi siempre para allá, como en quinto. De hecho, ahí también me sentí pasado a llevar. De hecho el mismo psicólogo me dijo, que yo siempre he sido así como inquieto, pero ellas pensaban que yo tenía así como déficit atencional, y el colegio, iba a psicólogos, y los psicólogos quedaban así como... no tenía nada sí. Hablar con una persona, no me distrae, puedo estar en una conversación, pero siempre he sido como inquieto en grupos. En mi curso siempre he sido como el más inquieto, y ahí una profe que fue mi 
profe jefe de primero hasta cuarto, ella fue como que me mandó a psicólogo. Mi mamá como que igual se sintió pasada a llevar." (Entrevista 7, P.4)

Como puede observarse en la cita anterior, los apoyos educativos que el establecimiento brinda son de carácter segregador, pues separan a quienes requieren de ayuda de los que no (Peña, 2013; Murillo y Duk, 2016). Ello subdivide a los miembros del grupo en pequeños subgrupos dentro de la sala de clases, lo que es resentido por el estudiantado. Esta separación lleva consigo un estigma reconocido por el estudiantado, que afecta al menos su dimensión emocional. Ello profundiza los perjuicios de la segregación en el aprendizaje que se ha descrito. (Treviño, et al., 2018)

Esta percepción de que se trata de un grupo minoritario queda aún más en evidencia a través del relato del estudiantado participante, cuando mencionan constantemente a quienes no cumplen las expectativas como miembros de un grupo pequeño (ver cita 13 y 14). Además, identifican a este grupo como un par de compañeros o compañeras se aíslan al final de la sala, lo que deja en evidencia el recién mencionado estigma.

Cita 13: "En mi curso hace dos años habían dos personas que siempre se sentaban solas al final, pero, era por gusto propio, no se llevan, no se llevaban con nadie del curso, no se querían incluir en ningún lado. El profe les decía y no hacían nada." (Entrevista 1, P.5).

Cita 14: “...yo no sabría decirlo, igual los profesores tratan de incluirlo más, por eso me gustan los trabajos grupales, para incluir más a esa persona en el grupo. En una de esas, que también podría participar la psicóloga, para saber si es que tiene algún problema más como grave, o más como grave o como más complicado de aprendizaje." (Entrevista 3, P. 6).

En la caracterización que se hace de este último grupo como aquellos que no se quieren incluir, se encuentra el alumnado que ha sido etiquetado con necesidades educativas especiales (NEE). Este grupo es caracterizado como poseedor de un comportamiento diferente que no parece concordar con lo común. Resulta interesante cómo se caracteriza esto como una decisión personal, restando responsabilidad a la comunidad educativa. Esta cita es ilustrativa respecto de la falta de discusión sobre diversidad. El estudiantado habla sobre sus 
pares y su comportamiento no deseado, lo que anula la responsabilidad del contexto en dicho fenómeno.

Asimismo, frente al aislamiento, la práctica de respuesta utilizada por la institución es la normalización y justificación de la exclusión (ver cita 15 y 16). Esto ocurre especialmente cuando no afecta directamente al grupo mayoritario, o bien, cuando la inclusión puede perjudicar el rendimiento personal de los miembros de ese grupo. Aquello puede observarse cuando profesores y estudiantes deciden no actuar frente al aislamiento, lo que impide que el alumnado aislado participe activamente del grupo.

Cita 15: "Es que a ver, en ese sentido los profes son como mucho más dejados. Dentro de la sala de clases, si los profes te ven como más aislados, los profes te preguntan cosas, pero de ahí, te puedes incluir con él, o te puedes sentar acá, no. Como que muy pocos pasan de esa barrera." (Entrevista 4, P, 5)

Cita 16: "Es como complicado, porque tú ves que está aislada la persona, pero como no dicen, no se puede hacer tanto al respecto. Como que los mismos compañeros intentan integrar a la persona, el mismo profe igual, pero es difícil." (Entrevista 2, P. 4)

Así, la inclusión se ve obstaculizada por valores excluyentes (Booth y Ainscow, 2015), como el conformismo, desconfianza e individualismo. Este tipo de valores son identificados, para efectos de este estudio, como obstaculizadores. Ello desincentiva que el alumnado quiera incluir a otros en sus grupos, lo que niega la participación efectiva (Escribano y Martínez, 2013). Incluso cuando este grupo minoritario ya forma parte de un equipo de trabajo, prima la desconfianza puesto que se prioriza la obtención de buenas calificaciones por sobre la inclusión. En este sentido, el rendimiento académico aparece como un bien altamente valorado que se superpone al bienestar de la comunidad.

La responsabilidad de los valores obstaculizadores expuestos en los resultados no puede adosarse únicamente al alumnado, sino que se funda en un sistema educativo altamente competitivo que premia el desempeño personal, cuyo nivel de segregación se extiende a todos los niveles. La responsabilidad de la promoción de estos valores recae en quienes diseñan el sistema, pero también en quienes lo ejercen en el cotidiano en los centros educativos. Esto ocurre pues la competencia originada por las inequidades sociales de los estudiantes (López Melero, 2018) provoca la necesidad de superar al otro para sobrellevar 
estas desigualdades estructurales. Estas desigualdades del sistema educativo son reflejo de un sistema económico de libre mercado, que premia a quien supera la oferta de su contrincante (Gárate, 2019).

En el mismo sentido, la calificación facilita la exclusión y segregación, pues promueve el individualismo y desconfianza entre compañeros. A la vez, anula valores que facilitan la inclusión, como la cooperación, compañerismo y altas expectativas (ver cita 17 y 18).

Cita 17: "Me complica el trabajo en grupo porque tengo una manía, que es que quiero saber todo el rato cómo está avanzando el trabajo. Entonces para eso, si hay mucha gente, primero sé que hay gente que no va a hacer nada, y segundo tengo que dispersarme en muchos lugares y no me concentro mucho en mi parte." (Entrevista 8, P. 6)

Cita 18: "Lo promueven haciéndonos elegir nuestras parejas o nuestros grupos para trabajar. Igual, yo en mi, en lo que yo a mí respecta, me gusta más trabajar solo en las actividades como diapositivas y esas cosas, porque así no tengo que preocuparme de que otra persona tenga que hacer su parte del trabajo para que tenga buena nota" (Entrevista 3, P.3)

El efecto facilitador de la exclusión y segregación que ejerce la calificación queda en evidencia cuando el profesorado planifica actividades cooperativas. En ellas, la interdependencia de calificaciones entre pares obliga al alumnado a priorizar la obtención de una buena nota, sobre la inclusión o participación igualitaria del grupo. Ello ha quedado de manifiesto en los resultados de la investigación, como se pudo observar en la cita 6 . Incluso cuando el alumnado es integrado en grupos de trabajo, la expectativa con respecto a su rendimiento es baja, lo que merma su participación. Este resultado es relevante, pues se ha demostrado que el efecto de la calificación en la mediación docente (Segura Castillo, 2018) obstaculiza los apoyos que puede brindar el profesor a su alumnado. Por tanto, la utilización de la calificación tiene implicancias en la participación efectiva del estudiantado en el aula.

En este sentido, el estudiantado identifica la aparición de un tipo de profesional denominado profesional de apoyo. Estos suelen ser psicólogos, cuyo rol se reduce a definir diagnósticos, encontrar problemas y solucionarlos, enfocados específicamente en las denominadas NEE (Salum Tomé, 2020). Esta percepción consolida la idea de que el aprendizaje de algunos estudiantes es inmodificable (López, Echeita y Martín, 2009). 
Esta perspectiva explica por qué el estudiantado se ofende cuando son derivados a este tipo de profesionales de apoyo. Como queda en evidencia en la cita 7, el diagnóstico es una herramienta de segregación de aquellos que son etiquetados gracias a dichos diagnósticos. El mecanismo a través del cual se ejerce dicha respuesta es la creación de instrumentos de evaluación diferentes para este grupo. En este sentido, la práctica tiene un efecto socioeducativo sobre el aprendizaje, así como sobre las relaciones.

El mecanismo institucional que se reconoce como natural en la percepción del estudiantado, es que el bajo rendimiento debe explicarse en causas internas del estudiante y no en el contexto. De esta forma, la normalización y justificación de la exclusión se sustenta en los valores obstaculizadores de la inclusión. Ello pues la desconfianza e individualismo que se manifiesta en el alumnado les lleva a culpabilizar a sus pares de su situación, al considerar que son ellos quienes no se incluyen en el grupo. Del mismo modo, se responsabiliza a ese mismo grupo de estudiantes y sus familias por su falta de hábitos de estudio y rendimiento escolar (ver cita 19 y 20), puesto que el alumnado participante, manifiesta que ese tipo de actitudes provienen del hogar. Es así como les quitan a la institución y a sus integrantes la responsabilidad de incluir efectivamente la diversidad dentro del aula, para poner dicha responsabilidad en el estudiantado que consideran diferentes.

Cita 19: "Es que el problema de que cada uno aprenda, viene más o menos de la educación que reciben en la casa. A cada uno se le inculca la cultura de la educación y del estudio." (Entrevista 3, P. 5)

Cita 20: “....es que igual hay compañeros que no quieren aprender y ya ahí nadie puede hacer nada". (Entrevista 8, P. 4)

De esta forma, las políticas de respuesta a la diversidad funcionan de manera selectiva al segregar y dejar la responsabilidad del logro educativo de este grupo minoritario en los profesionales de apoyo. Ello desplaza la responsabilidad del profesorado en la formación de estos jóvenes, al desviarla hacia otros profesionales (López, et al., 2009; Parra Muñoz, et al., 2020).

Es así como el estudiantado entrevistado identifica una práctica que se relaciona con el rendimiento, que es la derivación del profesorado hacia un profesional de la educación que realiza evaluaciones para explicar este bajo rendimiento. Esta práctica es descrita como una 
negación de sus capacidades (ver cita 7), lo que deja en evidencia las bajas expectativas que el alumnado tiene sobre aquellos compañeros que son sujetos de esta segregación.

A través de esta práctica de evaluación, con motivo del bajo rendimiento, el alumnado es etiquetado decidiendo si forma o no parte de estos subgrupos. Su rol de estudiante se condiciona. Ello ocurre al ser una persona físicamente integrada en el aula, pero simbólicamente segregada, al ser identificada como parte de un grupo en desventaja que requiere de apoyos especializados.

Esto deja en evidencia que la diversidad es atendida de manera reactiva frente al fracaso escolar, pero no es considerada desde el diseño original de la institución. Esto pues la escuela está pensada para el grupo mayoritario de estudiantes que cumplen con las expectativas académicas y actitudinales. La comodidad que ofrece esta perspectiva de que la víctima de exclusión o cualquier otro tipo de vulneración tiene la responsabilidad de ello, es propia de una mirada retrospectiva que evade la responsabilidad (Giner Alegría, 2011).

Además, la ya mencionada competencia por obtener buenas calificaciones es incentivada por quienes planifican el sistema al utilizar la calificación como un premio transformado en puntaje o nota. Ello aumenta la posibilidad de que el estudiantado acceda a la educación superior, especialmente a aquellas carreras y universidades que son símbolo de futuro éxito laboral, y por tanto, bienestar personal. Así, el alumnado se enfoca en obtener buenas calificaciones para optar a mejores oportunidades, mientras que el profesorado se enfoca en que sus estudiantes logren buenos resultados que les permitan optar a estas oportunidades. En esta dinámica, la competencia y el individualismo es la norma, lo que aleja la inclusión del foco prioritario de trabajo. Esto, especialmente por el contexto del sistema educativo escolar, que premia el desempeño personal (Jarpa-Arriagada y Rodríguez-Garcés, 2017).

Otro de los mecanismos a través de los cuáles se normaliza y justifica esta exclusión es el conformismo del personal docente. Este conformismo se puede identificar en la poca participación del grupo minoritario de estudiantes en el logro de sus aprendizajes, lo que obstaculiza su inclusión (ver cita 21 y 22). Este obstaculizador predominará en tanto no se profundice en las diversidades que han provocado la baja participación del estudiantado minoritario, al atribuir nuevamente la responsabilidad de la exclusión a quienes son excluidos. 
Cita 21: “...siempre están los compañeros que no hacen nada, que es la gran mayoría en mi curso. Y el profe les llama la atención, las reta, pero sigue igual la clase." (Entrevista 3, P.1)

Cita 22: “...es que igual tengo compañeras que no se quieren integrar, andan como siempre solas, no hacen ningún esfuerzo por ser parte del grupo". (Entrevista 5, P.7)

Así, en relación con los resultados anteriormente expuestos, los procesos de normalización de la exclusión, segregación e integración no se dan de manera independiente. Por el contrario, interactúan en una dinámica compleja que tiene diversos efectos en el aula y que acrecientan estos tipos de respuestas. Se puede inferir que estos tres tipos de respuesta a la diversidad se encuentran normalizados, pues están arraigados en la dinámica escolar dentro del aula, al manifestarse en los valores obstaculizadores de la inclusión que fueron antes discutidos. De esta forma, la cultura escolar de la institución acepta que algunos estudiantes no participen, no conviva, no aprendan o simplemente no formen parte de la mayoría dominante, sin cuestionar dicha realidad.

\section{Conclusiones}

A partir de estos resultados es posible concluir que se identifica, en la percepción del estudiantado, una cultura inclusiva en un estado de avance significativo en esta comunidad. Esto, pues los valores facilitadores, políticas y estrategias de enseñanza inclusivas de las que dan cuenta permiten ver que la institución se caracteriza por un perfil que responde a la valoración de la diversidad presente en ella. Del mismo modo, fue posible identificar, a través de las percepciones del alumnado, obstaculizadores del proceso de inclusión, los cuales derivan de valores promovidos por un sistema altamente competitivo, estructurado y estandarizado (Lerena Romero y Trejos Guerra, 2015) que trae como consecuencia diversas acciones de exclusión, segregación e integración para determinados grupos de estudiantes.

Del mismo modo, las prácticas de respuesta en el aula necesitan de estrategias pedagógicas que fomenten la participación activa del aprendizaje en todas y todos, adecuando esas estrategias a las diversidades presentes en la sala. Por ello, es necesario seguir trabajando en la promoción del trabajo colaborativo controlando, como se ha sugerido en esta 
investigación, el rol que la calificación derivada de la evaluación sumativa tiene en este proceso.

La percepción de la respuesta a la diversidad por parte del alumnado da cuenta de una institución que resulta acogedora y creadora de identidad en sus estudiantes. Esta percepción de escuela la hace ser percibida como un lugar seguro para la expresión de la diversidad. Sin embargo, la respuesta a la diversidad se percibe en ausencia de discusión y análisis de las formas de exclusión, generando esto que valores y prácticas segregadoras se instalen en la cultura escolar. Estas prácticas y valores están relacionados con el rendimiento, la competencia y el individualismo.

De esta forma, las propuestas para avanzar en la conformación de una comunidad inclusiva deben centrarse en la necesidad de hacer de las diversidades presentes en la comunidad un tema de conversación constante. El fomento de valores que faciliten la inclusión debe darse en este contexto de discusión, reflexionando sobre responsabilidades individuales y colectivas en el logro del objetivo común que se plantea ser una comunidad inclusiva.

A partir de los resultados de investigación, su revisión y discusión, se proponen cuatro prácticas de respuesta a la diversidad. En su conjunto, actúan de manera dinámica para visibilizar y desarticular la exclusión, segregación e integración respectivamente.

En primer lugar es necesario incorporar al enfoque curricular del establecimiento un análisis y valoración de la diversidad, lo cual debiera quedar explícito en el proyecto educativo institucional. Esta práctica permitiría fortalecer la cultura inclusiva a través de la inserción transversal de este eje temático en todas las asignaturas (Vega Fuente et al., 2013), concretando la reflexión sobre las implicancias que las prácticas tienen en la vida cotidiana.

En segundo lugar, y para disminuir la presencia de valores como el individualismo, la desconfianza y el conformismo, se recomienda insertar estrategias de aprendizaje colaborativo con foco en el enriquecimiento de los objetivos de aprendizaje (Johnson y Johnson, 2018) más que en la evaluación sumativa de los desempeños esperados. Se espera que la interdependencia promovida por el trabajo colaborativo desarticule prácticas de exclusión, segregación e integración.

Una tercera recomendación busca dar respuesta al efecto que produce la calificación sobre el escenario socio escolar y que, como se ha visto, logra acrecentar los valores obstaculizadores por medio de la competencia (López Melero, 2018). Se propone democratizar la calificación, lo cual se materializa en que la construcción de los instrumentos de evaluación son co-construidos entre docentes y estudiantes. De esta forma, se complementan o se 
agregan las autoevaluaciones y coevaluaciones formativas, así como calificadas. El fomento de este tipo de estrategias colaborativas de aprendizaje debe estar acompañada, entonces, de una diversificación de instancias de evaluación formativa que anulen la calificación como un factor que intervenga en los grupos (Segura Castillo, 2018).

En cuarto lugar, se propone modificar la percepción que el alumnado tiene respecto del equipo profesional que realiza apoyo pedagógico. Así, se sugiere que la ejecución del currículo tenga un enfoque universal, lo cual implica que profesionales de la educación, independientemente de su función, ejerzan su acción en la sala de clases y dentro del diseño curricular definido (López, et al., 2009; Parra Muñoz, et al., 2020). De esta forma, se visibiliza el rol educativo de profesionales de la educación diferencial y de los programas de integración.

En su conjunto, estas cuatro propuestas buscan presentar una alternativa de prácticas de respuesta a la diversidad. Se busca con ellas, que rompan con los obstaculizadores que frenan el progreso de un proyecto educativo basado en la inclusión, lo que puede servir de base para nuevas investigaciones en la materia.

Es necesario clarificar que la investigación realizada solo tuvo la posibilidad de realizarse con un pequeño grupo de estudiantes que logró sortear los desafíos de la escolarización, por lo que se trata de una mirada reducida de la realidad. En este sentido, nuevas investigaciones buscarán enfocarse en aquellos y aquellas estudiantes que no lograron culminar sus estudios en el sistema de educación regular, producto de su exclusión y deserción.

La presente investigación llevó a comprender, desde la percepción del alumnado, cómo esta comunidad educativa responde a sus diferencias. Las prácticas excluyentes y segregadoras identificadas en esta investigación podrían contribuir a la comprensión del fenómeno de la deserción escolar, abriendo nuevos campos de investigación, por ejemplo, ¿cuál es el perfil de los estudiantes que abandonan el sistema escolar regular en Chile, sus diversas regiones y territorios específicos?, ¿cómo podemos evitar ese abandono? Al conocer la respuesta a esta pregunta, las prácticas de inclusión podrían constituir una respuesta sustancial en la reducción y prevención de la deserción. En un país en el que el abandono del sistema escolar regular es un tema contingente es de vital importancia develar cómo actúan y se superponen las prácticas de segregación y exclusión en los establecimientos, con el fin de impulsar políticas de retención escolar más eficaces. 


\section{Agradecimientos}

Agradecemos a la Agencia Nacional de Investigación y Desarrollo (ANID) por el financiamiento otorgado a través de la Beca de Magíster en Chile para Profesionales de la Educación, adjudicación 2018. Sin duda, el apoyo que prestan a quienes comienzan a desarrollarse en la investigación, es fundamental para construir una trayectoria en la academia.

\section{Referencias}

Alba Pastor, Carmen. (2015). Aportaciones del Diseño Universal para el Aprendizaje y de los materiales digitales para el logro de una enseñanza accesible. Recuperado de https://web.ua.es/es/accesibilidad/documentos/cursos/ice/dua-y-materiales-digitales.pdf

Barría Rojas, Solange. (2019). La formación docente y la práctica educativa del profesor para la inclusión sociolaboral en jóvenes con discapacidad intelectual. Análisis del contexto chileno. Perspectiva Educacional, 58(1), 121-146. Doi https://dx.doi.org/10.4151/07189729-vol.58-iss.1-art.780

Booth, Tony. y Ainscow, Mel. (2015). Guía para la educación inclusiva: desarrollando el aprendizaje y la participación en los centros escolares. FUHEM, OEI. http://repositorio.minedu.gob.pe/handle/20.500.12799/4642

Casanova, María. Antonia. (2020). Miradas de futuro: educación inclusiva para la sociedad democrática. Una revisión en el tiempo. Avances En Supervisión Educativa, (33). Doi https://doi.org/10.23824/ase.v0i33.683

Castillo Riquelme, Víctor, Rodríguez Garcés, Carlos y Escalona Burgos, Juan. (2018). Participación, vida democrática y sentido de pertenencia según tipo de establecimiento educativo en Chile. Páginas de educación, 11(2), 108-129. https://doi.org/10.22235/pe.v11i2.1630

Dávila Newman, Gladys. (2006). El razonamiento inductivo y deductivo dentro del proceso investigativo en ciencias experimentales y sociales. Laurus, 12(Ext), 180-205. Recuperado de https://www.redalyc.org/pdf/761/76109911.pdf

Decreto $N^{\circ} 170$. Fija normas para determinar los alumnos con necesidades educativas especiales que serán beneficiarios de las subvenciones para educación especial. (2009). Recuperado de https://especial.mineduc.cl/wp-content/uploads/sites/31/2018/06/DTO170 21-ABR-2010.pdf

Decreto $N^{\circ}$ 83. Aprueba criterios y orientaciones de adecuación curricular para estudiantes con necesidades educativas especiales de educación parvularia y educación básica. (2015). Recuperado de http://bcn.cl/2aboe

Escribano, Alicia., y Martínez, Amparo. (2013). Inclusión Educativa y Profesorado Inclusivo. Aprender juntos para aprender a vivir juntos. Recuperado de https://cutt.ly/IQvOnIO 
Florez, María Teresa. (2015). Validity and equity in educational measurement: The case of SIMCE. Psicoperspectivas, 14(3) 31-44. Recuperado de http://scielo.conicyt.cl/pdf/psicop/v14n3/art04.pdf

Figueroa-Céspedes, Ignacio, Soto, Jorge yYáñez-Urbina, Cristopher. (2019). Concepciones sobre el cambio educativo de docentes participantes de un proyecto de desarrollo inclusivo. Revista Educación, 43(1). doi: https://doi.org/10.15517/revedu.v43i1.31297

Gárate, Francisco Javier. (2019). Inclusión educativa en Chile: Un camino político-histórico con una ruta de empedrados, curvas y colinas. Revista Estudios en Educación, 2(2). 143167. Recuperado de http://ojs.umc.cl/index.php/estudioseneducacion/article/view/53

Giner Alegría, César Augusto. (2011). Aproximación psicológica de la victimología. Revista derecho y criminología, 25-54. Recuperado de ttps://cutt.ly/KQvOHGt

Grácia, Marta., Sanlorien, Pilar. y Segués, María Teresa. (2017). Motivación, afectos y relaciones en el aula inclusiva. Recuperado de https://cutt.ly/gQvOCrv

Gimeno Sacristán, José. (2011). La educación obligatoria: Una escolaridad igual para sujetos diferentes en una escuela común. En José Gimeno Sacristán, Rafael Feito Alonso, Philipe Perrenoud, y María Clemente Linuesa (Compiladores), Diseño, desarrollo e innovación del currículum (pp. 48-66). Madrid: Morata. Recuperado de https://cutt.ly/KQvONRF

González-Monteagudo, José. (2000). El paradigma interpretativo en la investigación social y educativa: Nuevas respuestas para viejas interrogantes. Cuestiones pedagógicas: Revista de ciencias de la educación, (15) 227-246. Recuperado de https://dialnet.unirioja.es/servlet/articulo?codigo $=625605$

Gurdián-Fernández, Alicia. (2007). El paradigma cualitativo en la investigación socioeducativa. Colección: Investigación y Desarrollo Educativo Regional. Recuperado de https://web.ua.es/es/ice/documentos/recursos/materiales/el-paradigma-cualitativo-enla-investigacion-socio-educativa.pdf

Hernández Soto, Nadia. (2016). Reflexión teórica sobre la Declaración de Incheon Educación 2030 "Hacia una educación inclusiva y equitativa de calidad y un aprendizaje a lo largo de la vida de todos". Revista nacional e internacional de educación inclusiva, 9(2) 18-36. Recuperado de https://revistaeducacioninclusiva.es/index.php/REl/article/view/48/44

Inostroza, Fabián. y Falabella, Alejandra. (2021). Educadoras diferenciales en Chile frente a las políticas de rendición de cuentas: Incluir, estandarizar y obedecer. Revista Mexicana de Investigación Educativa, 26(88). 123-148. Recuperado de http://www.researchgate.net/publication/346955196

Jarpa-Arriagada, Carmen Gloria. y Rodríguez-Garcés, Carlos. (2017). Segmentación y Exclusión en Chile: El caso de los Jóvenes Primera Generación en Educación Superior. Revista Latinoamericana de Ciencias Sociales, Niñez y Juventud, 15(1), 327-343. Recuperado de http://revistaumanizales.cinde.org.co/rlcsnj/index.php/RevistaLatinoamericana/article/view/2966/797 
Johnson, Davis W., y Johnson, Roger T. (2018). Aprendizaje Cooperativo e inclusión. Impacto de la interdependencia social de los resultados. En Juan Carlos Torrego, Laura Rayón, Yolanda Muñoz y Patricia Gómez (Editores.), Inclusión y Mejora Educativa (pp. 19-47). Alcalá, España: Universidad de Alcalá. Servicio de publicaciones.

Lerena Romero, Bruno. y Trejos Guerra, Jimmy. (2015). Sobre la posibilidad de una educación inclusiva bajo el actual modelo de desarrollo económico y social chileno. Revista de Educación Inclusiva, 9(2). 145-160. Recuperado de https://dialnet.unirioja.es/servlet/articulo?codigo $=5504536$

Ley $N^{\circ}$ 19.284. Establece normas para la plena integración social de las personas con discapacidad. (1994). Ministerio de Planificación y Cooperación. Recuperado de https://www.leychile.cl/Navegar?idNorma=30651

Ley $N^{\circ}$ 20.422. Establece normas sobre igualdad de oportunidades e inclusión social de personas con discapacidad. (2010). Ministerio de Planificación. Recuperado de https://www.leychile.cl/Navegar?idLey=20422

Ley $N^{\circ}$ 20.845. De inclusión escolar que regula la admisión de los y las estudiantes, elimina financiamiento compartido y prohíbe el lucro en establecimientos educacionales que reciben aportes del estado. (2015). Ministerio de Educación. Recuperado de https://www.leychile.cl/Navegar?idNorma=1078172ybuscar=ley+20845

López, Mauricio., Echeita, Gerardo. y Martín, Elena. (2009). Concepciones sobre el proceso de inclusión educativa de alumnos con discapacidad intelectual en la educación secundaria obligatoria. Cultura y Educación, 21(4), 485-496. Doi https://doi.org/10.1174/113564009790002391

López Melero, Miguel. (2018). Fundamentos y prácticas inclusivas en el proyecto Roma. Madrid: MORATA.

Mayan, María J. (2001). Una introducción a los métodos cualitativos: Módulo de entrenamiento para estudiantes y profesionales. Edmonton: Qual Institute Press. Recuperado de http://www.ualberta.ca/ iiqm//pdfs/introduccion.pdf

Martín Ortega, Elena. y Mauri Majós, Teresa. (2011). Atención a la diversidad en la escuela inclusiva. En Elena Martín, y Teresa Mauri (Eds.), Orientación educativa. Atención a la diversidad y educación inclusiva (pp. 29-49). Barcelona: Graó. Recuperado de https://cutt.ly/lQv0901

Mella Valenzuela, Orlando. y Osses Bustingorry, Sonia. (2015). Métodos y técnicas introductorios a la investigación científica en ciencias sociales y educación. Temuco: Ediciones Universidad de La Frontera.

Moliner García, Odet. (2013). Educación Inclusiva. Castellón de la Plana: Unión de Editoriales de Universidades Españolas. Recuperado de http://repositori.uji.es/xmlui/bitstream/handle/10234/72966/s83.pdf?sequence=1 
Muñoz Morán, Cristina Aracely. (2018). Prácticas Pedagógicas en el Proceso de Transición hacia la Escuela Inclusiva. Seis experiencias en El Salvador. Revista Latinoamericana de Educación Inclusiva. 12(1), 95-110. Recuperado de https://scielo.conicyt.cl/pdf/rlei/v12n1/0718-7378-rlei-12-01-00095.pdf

Murillo, F. Javier. y Duk, Cynthia. (2016). Segregación Escolar e Inclusión. Revista Iberoamericana de Educación Inclusiva, 10(2), 11-13. Recuperado de https://scielo.conicyt.cl/pdf/rlei/v10n2/art01.pdf

Naciones Unidas, Cepal. (2007). Panorama Social de América Latina 2007. UNFPA. Recuperado

de https://www.cepal.org/sites/default/files/publication/files/1227/S0700764 es.pdf

Navarro-Aburto, Braulio Ademir., Arriagada Puchel, Inés Analía., Osses-Bustingorry, Sonia. y Burgos-Videla, Carmen Gloria. (2016). Adaptaciones curriculares: Convergencias y divergencias de su implementación en el profesorado chileno. Educare, 20(1), 1-18. DOI: https://doi.org/10.15359/ree.20-1.15

Núñez-Muñoz, Carmen Gloria., Peña-Ochoa, Mónica., González-Niculcar, Bryan. y AscorraCosta, Paula. (2020). Una mirada desde la inclusión al Programa de Integración Escolar (PIE) en escuelas rurales chilenas: un análisis de caso. Revista Colombiana de Educación, 1(79), 347-368. DOI: https://doi.org/10.17227/rce.num79-9725

Ortiz Jiménez, Luis., López Meneses, Eloy., Figueredo Canosa, Victoria. y Martín Padilla, Antonio Hilario. (2018). Diversidad e inclusión educativa. Respuestas innovadoras con apoyo en las TIC. Barcelona: Editorial Octaedro. Recuperado de https://octaedro.com/wp-content/uploads/2019/02/16123.pdf

Parra Muñoz, Hugo., Garrido Reyes, Catalina., Carrasco Lobos, Andrea., Vergara Rodríguez, Mauricio., Hidalgo Kawada, Felipe. y Meza Buzzetti, David. (2020). Normocentrismo, diversidad y alteridades: Deconstruyendo las políticas educativas inclusivas en Chile. Archivos Analíticos de Políticas Educativas, 28(100). DOI: https://doi.org/10.14507/epaa.28.4338

Peña, Mónica. (2013). Análisis crítico de discurso del Decreto 170 de Subvención Diferenciada para Necesidades Educativas Especiales: El diagnóstico como herramienta de gestión. Psicoperspectivas, 12(2), 93-103. Recuperado de https://scielo.conicyt.cl/pdf/psicop/v12n2/art10.pdf

Poblete-Christie, Octavio, López, Mauricio y Muñoz, Loreto. (2019). ¿De la frustración a la alegría y de la alegría a la frustración? Significados sobre inclusión educativa construidos por docentes a partir de su experiencia emocional. Psykhe, 28(1) 1-14. DOI: https://doi.org/10.7764/psykhe.28.1.1126

Queupil, Juan Pablo. y Durán del Fierro, Francisco. (2018). El Principio de Inclusión: Similitudes y Diferencias en la Educación Escolar y Superior en Chile. Revista latinoamericana de educación inclusiva, 12(1), 111-128. Doi https://dx.doi.org/10.4067/S0718-73782018000100111 
Ramírez Valbuena, Wílmar Ángel. (2017). La inclusión: una historia de exclusión en el proceso de enseñanza-aprendizaje. Cuadernos de Lingüística Hispánica, (30), 211-230. Doi https://doi.org/10.19053/0121053X.n30.0.6195

Rittaco Real, Maximiliano. y Amores Fernández, Francisco Javier. (2012). Prácticas escolares ante la exclusión social. Estudio en la educación secundaria obligatoria. Contextos Educativos, (15), 41-60. Recuperado de https://publicaciones.unirioja.es/ojs/index.php/contextos/article/view/654/617

Salum Tomé, José Manuel. (2020). Inclusión escolar en el marco de la nueva normativa legal en Chile. Brazilian Journal of Development, 6(6), 39244-39262. DOI: https://doi.org/10.34117/bjdv6n6-461

Segura Castillo, Mario Alberto (2018). La función formativa de la evaluación en el trabajo escolar cotidiano. Revista Educación, 42(1), 118-137. DOI https://doi.org/10.15517/revedu.v42i1.22743

Sinisi, Liliana. (2010). Integración o inclusión escolar: ¿Un cambio de paradigma? Boletín de Antropología $\quad y \quad$ Educación, $\quad$ (1), 11-14. http://antropologia.institutos.filo.uba.ar/sites/antropologia.institutos.filo.uba.ar/files/bae $\underline{\text { n01a02.pdf }}$

Strauss, Anselm. y Corbin, Juliet. (2012). Bases de la investigación cualitativa. Antioquia: Universidad de Antioquia. Recuperado de https://cutt.ly/oQvO5IU

Treviño, Ernesto., Valenzuela, Juan Pablo., Villalobos, Cristóbal., Béjares, Consuelo., Wyman, Ignacio. y Allende, Claudio. (2018). Agrupamiento por habilidad académica en el sistema escolar. Revista Mexicana de Investigación Educativa, 23(76), 45-71. Recuperado de http://www.scielo.org.mx/pdf/rmie/v23n76/1405-6666-rmie-23-76-45.pdf

Torrego, Juan Carlos., Monge, Carlos., Pedrajas, Mercedes Lorena. y Martínez Virseda, Concepción. (2015). Formación del profesorado en aprendizaje cooperativo y alumnos con altas capacidades: Un enfoque inclusivo. Revista latinoamericana de educación inclusiva, 9(2), 91-110. Recuperado de https://dialnet.unirioja.es/servlet/articulo?codigo $=5504538$

Unesco. (2011). IV Jornadas de Cooperación Iberoamericana sobre Educación para la Paz, la Convivencia Democrática y los Derechos Humanos. Santiago: OREALC/UNESCO/SANTIAGO. Recuperado de https://unesdoc.unesco.org/ark:/48223/pf0000191613?pos InSet=7yqueryld=28b00f32fa66-4aca-9905-60af3ba063e4

Vázquez-Recio, Rosa. y López-Gil, Mónica. (2018). Interseccionalidad, jóvenes "sin sistema" y resistencia. Una mirada diferente del fracaso/abandono escolar. Revista Brasileira de Educación, 23, 1-24. Doi https://doi.org/10.1590/s1413-24782018230094 
Vega Fuente, Amando., López Torrijo, Manuel. y Garín Casares, Segundo María. (2013). Educación inclusiva. Entre la crisis y la indignación. Intersticios Revista Sociológica de Pensamiento Crítico, 7(1), 315-336. https://dialnet.unirioja.es/servlet/articulo?codigo $=4352164$

Véliz Jorquera, Paulina., Martínez, María Jesús., Parra Muñoz, Hugo. y Garrido Reyes, Catalina. (2020). Integración, inclusión y justicia social: Reflexiones desde las normativas inclusivas en la educación chilena. Revista Actualidades Investigativas en Educación, 20(2). 1-28. DOI https://doi.org/10.15517/aie.v20i2.41709 


\section{Revista indizada en}
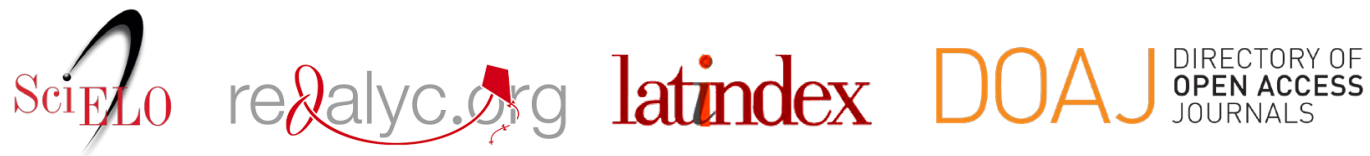

Distribuida en las bases de datos:
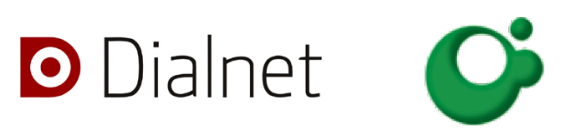
SHERPA/RøMEO

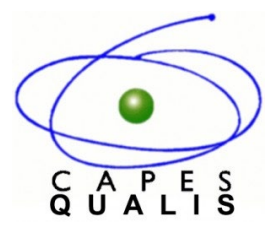

MIAR 